\title{
REPRODUCTION
}

REPRODUCTION

\section{INDUCTION D'UNE GESTATION PENDANT LA LACTATION. COMPARAISON DE DEUX MOMENTS D'INJECTION DE PMSG}

\author{
F. BOTTE-MARTINAT, F. DU MESNIL DU BUISSON, \\ F. BARITEAU et P. MAULÉON \\ Station de Physiologie de la Reproduction, \\ Centre de Recherches de Tours, I. N. R. A., \\ B. P. 1, Nouzilly, 37380 Monnaie
}

\section{RÉSUMÉ}

Cent soixante et une truies multipares Large White, Landrace ou croisées appartenant à des éleveurs ont été soumises à un traitement hormonal pendant la lactation. Une injection de 2 ooo UI de PMSG est effectuée, soit le $\mathrm{I} 8 \pm 2$ jours $\left(\mathrm{MB}_{18}\right)$, soit le $32 \pm 2$ jours après la mise bas $\left(\mathrm{MB}_{32}\right.$; $\mathrm{MB}_{0}$ étant le jour de la mise bas). Les femelles subissent une double insémination le $4^{\mathrm{e}}$ et le $5^{\mathrm{e}}$ jour après l'injection de PMSG qu'elles soient en cestrus ou non. L'éleveur décide de la date de tarissement de la truie.

Parmi les I6r truies traitées, 69 (soit 42,8 p. roo) ont mis bas en moyenne 9,2 porcelets par portée. Le moment d'intervention pendant la lactation modifie significativement le taux de réussite : $37 \mathrm{p}$. Ioo de truies pleines lorsque le traitement débute à $\mathrm{MB}_{18}$ contre $45,8 \mathrm{p}$, Ioo dans le cas où l'injection a lieu $\mathrm{I}_{4}$ jours plus tard.

Le taux de gestation induit, la prolificité tendent à diminuer si la taille de la portée allaitée passe de 5 à ro et plus. La durée de la lactation semble interférer avec le traitement proposé : pour le lot $\mathrm{MB}_{32}$, la fertilité est plus faible chez les truies qui ont allaité plus de 55 jours. L'irrégularité des retours en oestrus après le sevrage constitute un handicap : 73,6 p. Ioo des truies non pleines ont eu leur premier ostrus plus de ro jours après l'arrêt de la lactation.

\section{SUMMARY}

\section{INDUCTION OF PREGNANCY DURING LACTATION. COMPARISON OF TWO DATES FOR PMSG INJECTION}

One hundred and sixty one multiparous Large White, Landrace or crossbreed sows were subjected to a hormonal treatment during lactation. An injection of 2 ooo IU of PMSG was made, either on day $18 \pm 2\left(\mathrm{MB}_{18}\right)$, or on day $32 \pm 2$ after farrowing $\left(\mathrm{MB}_{32} ; \mathrm{MB}_{0}\right.$ being the day of farrowing). The females, in cestrus or not were subjected to double insemination the $4^{\text {th }}$ and 5 th day after the injection of PMSG.

Among the I6I sows treated, $69(42,8$ p. IOo) gave a mean number of 9,2 piglets per litter. 
The moment of intervention during lactation significantly modified the conception rate : $37 \mathrm{p}$. Ioo of pregnant sows when the treatment started at $\mathrm{MB}_{18}$ versus $45,8 \mathrm{p}$. roo when the injection was made 14 days later.

The induced gestation rate and the prolificacy tended to decrease if the size of the suckled litter increased from 5 to ro and more. The lactation length seemed to interfer with the treatment proposed : for group $\mathrm{MB}_{32}$, fertility was lower in the sows having fed their litter for more than 55 days. The irregularity in returns to oestrus after weaning constituted a handicap : $73.6 \mathrm{p}$. Ioo of the non pregnant sows showed their first oestrus Io days after ceasing of lactation.

\title{
INFLUENCE DE L'ALIMENTATION PENDANT LA CROISSANCE DE LA TRUIE SUR LA MATURITÉ SEXUELLE ET LES PERFORMANCES DE REPRODUCTION : RÉSULTATS PRÉLIMINAIRES
}

\author{
P.-H. DUÉE et M. ÉTIENNE \\ Station de Recherches sur l'Élevage des Porcs, \\ Centre national de Recherches zootechniques, I. N.R. A., \\ 78350 Jouy en Josas

\section{RÉSUMÉ}

Deux expériences ont été réalisées afin de déterminer les effets d'une restriction alimentaire d'une part, d'une carence en protéines et en lysine d'autre part pendant toute ou partie de la croissance, sur l'apparition de la puberté, le taux d'ovulation au premier cestrus et les performances de reproduction à 30 jours de gestation, les animaux ayant été saillis au premier oestrus.

La première expérience porte sur deux lots de I 2 Truies Large White soumises, à partir d'un poids vif de $55 \mathrm{~kg}$ et jusqu'à la puberté, soit à un niveau alimsntaire libéral (consommation maximale $3.28 \mathrm{~kg}$ par jour), soit à une restriction d'environ $45 \mathrm{p}$. Ioo du niveau précédent.

Une sous-alimentation pendant la croissance retarde de deux mois l'âge à la puberté des truies ( 363 contre 296 jours) et abaisse leurs poids à ce stade. De plus, les truies restreintes subissent une diminution du taux d'ovulation ( 12,0 contre ${ }_{13}, 8$ ) qui se répercute sur le nombre d'embryons viables à 30 jours de gestation $(9,0$ contre 12,5$)$. La mortalité embryonnaire apparaît plus élevée dans ce lot de truies restreintes avant la puberté (26,o contre $8,8 \mathrm{p}$. Ioo).

La seconde expérience compare trois lots de 9 truies, à partir de $25 \mathrm{~kg}$ de poids vif et jusqu'à la puberté, recevant suivant une échelle de rationnement, soit un régime à faible taux azoté ( I I, 5 p. I00) supplémenté ou non en lysine (teneur totale des régimes: lot $\mathrm{I}: 0,42 \mathrm{p}$. 100; lot 2 : $0,62 \mathrm{p}$. IоO) soit un régime bien pourvu en protéines (lot $3:$ matières azotées : 17,5 p. Ioo, lysine: o,86 p. I00).

Une restriction azotée retarde la croissance des truies (lot I), principalement pendant les trois premiers mois. Un supplément de L-lysine (lot 2) améliore cependant le gain moyen quotidien. L'apport complémentaire de protéines n'est bénéfique, sur le plan de la croissance, que pendant la première période (trois premiers mois). En ce qui concerne l'âge à la puberté, aucune différence significative n'est observée entre les lots (lot $I: 253$ jours, lot $2: 254$ jours, lot $3: 249$ jours). Par rapport aux autres lots, le poids à la puberté des truies du lot I est significativement plus faible; de même que le taux d'ovulation (lot $1 ; 9,8 ; \operatorname{lot} 2: 12,7 ; \operatorname{lot} 3: \mathbf{1} 3,0$ ). Cette diminution du taux d'ovulation se répercute sur la taille de la portée à 3 o jours (lot $1: 8,2 ; \operatorname{lot} 2: 9,7 ;$ lots $3: 10,0)$. 